\title{
EXPANDING MAPS ON SETS WHICH ARE ALMOST INVARIANT: DECAY AND CHAOS
}

BY

\author{
GIULIO PIANIGIANI AND JAMES A. YORKE ${ }^{1}$
}

\section{Dedicated to José Massera}

\begin{abstract}
Let $A \subset R^{n}$ be a bounded open set with finitely many connected components $A_{j}$ and let $T: \bar{A} \rightarrow R^{n}$ be a smooth map with $A \subset T(A)$. Assume that for each $A_{j}, A \subset T^{m}\left(A_{j}\right)$ for all $m$ sufficiently large. We assume that $T$ is "expansive", but we do not assume that $T(A)=A$. Hence for $x \in A, T^{i}(x)$ may escape $A$ as $i$ increases. Let $\mu_{0}$ be a smooth measure on $A$ (with inf ${ }_{A} d \mu_{0} / d x>0$ ) and let $x \in A$ be chosen at random (using $\mu_{0}$ ). Since $T$ is "expansive" we may expect $T^{i}(x)$ to oscillate chaotically on $A$ for a certain time and eventually escape $A$. For each measurable set $E \subset A$ define $\mu_{m}(E)$ to be the conditional probability that $T^{m}(x) \in E$ given that $x$, $T(x), \ldots, T^{m}(x)$ are in $A$. We show that $\mu_{m}$ converges to a smooth measure $\mu$ which is independent of the choice of $\mu_{0}$. One dimensional examples are stressed.
\end{abstract}

1. Introduction. Lorenz [4] was studying a system of differential equations depending on a parameter $R$. For small values of $R\left(1<R<R_{0} \cong 13.926\right.$ [1]) there are two stable and one unstable fixed points and all trajectoires go eventually to these points. For $R=28$ Lorenz observed chaotic trajectories. Kaplan and Yorke [1] and Robbins [6] observed a value $R_{1} \cong 24.06$ as transition point between the nonchaotic and the chaotic regions. If one approaches the value $R_{1}$ from below he observes trajectories that look chaotic for a certain time and then go to one of the fixed points. J. Yorke and E. Yorke [8] made extensive numerical investigations of this transition. We develop a theory which explains rigorously these observations. There is also a larger context into which this paper fits. There is a variety of phenomena in which trajectories appear chaotic for an extended period of time but then settle down. Consider a particularly difficult problem of this type. Picture an energy conserving billiard table with smooth obstacles so that all trajectories are unstable with respect to the initial data. Now suppose a small hole is cut in the table so that the ball can fall through. We would like to investigate the statistical behavior of such phenomena. In particular, suppose a ball is started

\footnotetext{
Received by the editors May 30, 1978 and, in revised form, August 10, 1978.

AMS (MOS) subject classifications (1970). Primary 28A65.

Key words and phrases. Frobenius-Perron operator, invariant measures, expanding maps, chaos.

${ }^{1}$ This research was partially supported by the National Science Foundation under grant MCS-76-24432.
} 
on the table in some random way according to some probability distribution. Let $p(t)$ be the probability that the ball stays on the table for at least time $t$ and let $p_{E}(t)$ be the probability that the ball is in a measurable set $E$ after time $t$. Does $p_{E}(t) / p(t)$ tend asymptotically to some constant $\mu(E)$ as $t$ goes to infinity? And if it does, what are the properties of $\mu$ ? Does it depend on the initial distribution? Unfortunately, we cannot answer these questions in general, but we do investigate a class of simpler situations that effectively includes the pre-turbulent Lorenz model for fluid flow. In our results the limiting $\mu$ is shown to be smooth, though in cases such as the billiard problem, this would not seem to be the case.

Assumptions are necessary to guarantee the existence of a smooth asymptotic measure $\mu$. Instability of solutions on initial data is closely related to good statistical behavior. For the one dimensional case, $T:[0,1] \rightarrow[0,1]$, piecewise smooth, Lasota and Yorke [2] proved the following result. Assume that $\inf \left|T^{\prime}(x)\right|>1$ for $x$ where $T^{\prime}$ is defined; then there exist absolutely continuous invariant measures (see also [3], [5], [9]). Notice that the condition $\inf \left|T^{\prime}(x)\right|=\lambda>1$ guarantees that all trajectories are unstable with respect to the initial data since for any $x$, if $y$ is close to $x$, then $|T(x)-T(y)|>\lambda \mid x-$ $y \mid$. In particular no periodic orbits can be stable.

In this paper we investigate maps $T: \bar{A} \rightarrow R^{n}$ where $A \subset R^{n}$ is open. We assume $T$ is "expansive" in a sense defined in the next section and in particular $A \subset T(A)$. In general $T(A)-A$ is nonempty and when $T(A) \neq A$ we will show that almost every $x$ is eventually mapped out of $A$ by some iterate of $T$. Suppose that the point $x$ is chosen in $A$ according to some initial probability measure $\mu_{0}$. Notice that $\mu_{0}\left(T^{-1}(A)\right)$ is the probability that $T(x) \in$ $A$. Write $\mu_{1}$ for the normalized measure given by

$$
\mu_{1}(E)=\mu_{0}\left(T^{-1}(E)\right) / \mu_{0}\left(T^{-1}(A)\right)
$$

where $E$ is a Borel set. We discuss problems in which these quantities are well defined. A measure $\mu_{0}$ is said to be conditionally invariant if $\mu_{1}(E)=\mu_{0}(E)$ for all Borel sets in $A$.

Our results extend without change to the case where $A$ is an open subset of an $n$ dimensional Riemannian manifold instead of $R^{n}$. Expansive maps have been investigated in [10] $[12]$ for cases where $T(A)=A$, where $A$ is sometimes an $n$ dimensional compact manifold.

Instead of asking questions about $\mu$, one can ask about the probability $p_{n}$ that $x \in A$ and $T^{i}(x) \in A$ for all $i=1, \ldots, n$. Specifically does $p_{n} \rightarrow 0$ exponentially as $n \rightarrow \infty$ ? That is, is there some $k<1$ for which $k^{-n} p_{n}$ converges to a constant as $n$ tends to $\infty$ ? In our case this is true with $k=\mu\left(T^{-1}(A)\right)$ where $\mu$ is the conditionally invariant measure. Bowen and Ruelle [14] have results which can be shown to imply exponential decay; see 
[14, Theorems 4.4a and 5.6] or [15, p. 197] and for the flow case see Franco-Sanchez [17, p. 499]. Ruelle and Bowen discuss the case where the map is a homeomorphism, but their results extend to more general maps in some cases, by taking inverse limits, turning the map into a homeomorphism on a bigger space. It will be of value to determine how broad the applicability is of the Bowen-Ruelle results.

While the pool table (that is a billiard table with holes) presents an interesting long term problem, another more speculative class of problems has provided a greater motivation to our work. These problems come from the study of climate and fluid dynamics. Many experiments with fluids are aimed at determining the steady state, yet an experiment may require substantial waiting time for it to settle down to an apparent steady state. We feel that the original state may often have been metastable. The decay time may be quite long and the experimenter does not know how long to wait for the final transition. Some experiments exhibit changes after the experiment had appeared to settle down to a final noisy state. We are led to suggest that such experiments are examples of metastable processes: after indeterminant periods, internally caused fluctuations may combine in some random way to cause the transition. The new resulting state may also be metastable. In particular the earth's climate may be metastable. Of course our climate is affected by regular variations in the earth's orbit and by changes in the constituents of the atmosphere. Metastability would imply that small random changes can combine to produce large long term changes. Accurate climate models will be exceedingly complex systems, if indeed they are ever developed. We have shown however that even the simplest nonlinear process exhibits metastability in certain parameter ranges (application (2)). Also a simple chaotic system of differential equations (the Lorenz system-see application (1)) can behave in a metastable manner. Metastability is always difficult to demonstrate, yet the suggestion remains that metastability may be lurking in far more complex systems.

2. Assumptions for expansive maps. Let $A=\bigcup_{i=1}^{p} A_{i}$ where $A_{i} \subset R^{n}$ are disjoint open connected sets. We also assume each $A_{i}$ is "arc-wise bounded" in the following sense: there is a number $\delta_{i}$ such that any two points in $A_{i}$ can be joined by a polygonal arc (i.e., piecewise straight arc) of length at most $\delta_{i}$. There will be a smallest such number which we call the arc diameter of $A_{i}$. Let $T: \bar{A} \rightarrow R^{n}$ and let $B=A \cap T^{-1}(A)$. We assume that $T$ is a twice differentiable mapping on $B$ and, on $B$, there is a uniform bound on all first and second partial derivatives. Notice that for all $x \in A, T^{-1}(x)$ is a uniformly finite set; i.e., $T^{-1}(x)$ contains at most $q$ points for some $q$ independent of $x$. We say a matrix $M$ is $\lambda$-expansive if $\inf \{|M \cdot v|:|v|=1\} \geqslant$ $\lambda$. We say the mapping $T$ is expansive if the following conditions are satisfied. 
E1. $A \subset T(A)$.

E2. $A \cap T(\partial A)$ is empty, where $\partial A$ is the boundary of $A$.

E3. There exists $\lambda>1$ such that $D T(x)$ is a $\lambda$-expansive matrix for all $x \in B$, where $D T(x)$ is the Jacobian matrix for $T$.

For a set $S \subset A$, define $T^{n}(S)=T\left(T^{n-1}(S)\right), n>1$. We say the mapping $T$ is transitive on components if for each pair $i, j, 1 \leqslant i, j \leqslant p$, there exists a positive integer $n=n(i, j)$ such that $A_{i} \cap T^{n}\left(A_{j}\right)$ is nonempty. In what follows $m$ is the Lebesgue measure and $C(A)$ is the set of all continuous functions from $A$ to $R$. Since $m(A)$ is finite, there is no loss in assuming $m(A)=1$.

3. Frobenius-Perron operator. For a given $f \in C(A)$ let $\mu_{f}$ be the measure defined by $d \mu_{f}=f d m$. We call $f$ the density of $\mu_{f}$. Let $\|\cdot\|_{1}$ be the $L^{1}$ norm and define

$$
P_{1}(f(x))=d\left(\mu_{f} \circ T^{-1}\right) / d m
$$

and if $\left\|P_{1}(f)\right\|_{1}>0$ define $P(f) \in L^{1}(A)$ by

$$
P(f(x))=P_{1}(f(x)) /\left\|P_{1}(f)\right\|_{1} .
$$

The Frobenius-Perron operator $P$ has the following properties:

(i) $f$ nonnegative everywhere on $A$ implies $P f$ is also.

(ii) $\int_{A} P(f) d m=1$.

(iii) $P f=f$ if and only if the measure $\mu$ defined by $d \mu=f d m$ is conditionally invariant, i.e., $\mu\left(T^{-1}(E)\right)=\alpha \mu(E)$ for all Borel sets $E$ where $\alpha$ is the constant $\mu\left(T^{-1}(A)\right)$.

For $x \in A, T^{-1}(x)$ is a finite set and if $y \in T^{-1}(x)$ we have $D T(y)$ is nonsingular. Hence there is a neighborhood $U$ of $x$ and local inverse functions $\varphi_{i}: U \rightarrow A$ such that $T \circ \varphi_{i}=$ id on $U$, and $T(y) \in U$ implies that for some $\varphi_{i}, \varphi_{i} \circ T(y)=y$. On $U, P$ has an explicit formula:

$$
P f(x)=\sum_{i}\left|\operatorname{det} D \phi_{i}(x)\right| f \circ \phi_{i}(x) / \int_{T^{-1}(A)} f d m .
$$

In the 1-dimensional case $U$ may be chosen to be the component of $A$ containing $x$, although in higher dimensions this is sometimes impossible. $T$ is not necessarily one-to-one even when $T$ is restricted to connected subsets of $T^{-1}(A)$ yet $U$ must be chosen so that $T$ is one-to-one. We leave it to the reader to remember that the set $\left\{\varphi_{i}\right\}$ is defined only on a neighborhood of $x$ and that while the number of functions in this set is finite, this number may be different for different components of $A$. From condition E2 it follows that the function $P f$ is continuous if $f$ is continuous. 
4. The existence and uniqueness theorems. We will need a simple observation.

LEMMA 4.1. For any vectors $a_{i}$ and positive real numbers $b_{i}, i=1, \ldots, q$, we have

$$
\left|\frac{\Sigma a_{i}}{\Sigma b_{i}}\right| \leqslant \max _{i}\left|\frac{a_{i}}{b_{i}}\right| .
$$

The proof follows immediately from the fact that $\Sigma a_{i} / \Sigma b_{i}$ is in the convex hull of $\left\{a_{i} / b_{i}\right\}$.

THEOREM 1. Let $T: \bar{A} \rightarrow R^{n}$ be expansive. Then there exists a measure $\mu$ such that:

(i) $\mu$ is conditionally invariant,

(ii) $\mu$ is absolutely continuous with respect to $m$,

(iii) $\mu(A)=1$.

Furthermore if $T \in C^{\infty}(\bar{A})$ then any $\mu$ which satisfies (i)-(iii) is in $C^{\infty}$ : that is the density $d \mu / d m$ is in $C^{\infty}(\bar{A})$.

Proof of Existence. (We delay the $C^{\infty}$ proof until the Appendix.) For all nonnegative Lipschitzean functions in $C(A)$ we define the "regularity" of $f$ to be

$$
\operatorname{Reg}(f)=\sup \left\{\left|f^{\prime}(x)\right| / f(x): x \in A \text { and } f^{\prime}(x) \text { is defined and } f(x)>0\right\}
$$

where $f^{\prime}$ means the gradient of $f ;|y|$ is the Euclidean norm of $y$. Define

$$
H=\left\{f \in C(A): f \geqslant 0, f \text { is Lipschitzean, } \operatorname{Reg} f<\infty \text { and } \int_{A} f d m=1\right\} .
$$

We claim that:

$$
\underset{n \rightarrow \infty}{\limsup } \operatorname{Reg}\left|P^{n}(f)\right| \leqslant \rho \quad \text { for all } f \in H
$$

where $\rho$ is independent of $f$. To evaluate $\operatorname{Reg}(P f)$, let $J_{i}(x)=\left|\operatorname{det} D \varphi_{i}(x)\right|$ and $\left\|D \varphi_{i}(x)\right\|=\sup _{|v|=1}\left|D \varphi_{i}(x), v\right|$. Using Lemma 4.1 we have:

$$
\begin{aligned}
\frac{\left\lfloor(P f)^{\prime} \mid\right.}{P f} & =\frac{\left|\sum_{i}\left[J_{i} f \circ \varphi_{i}\right]^{\prime}\right|}{\sum_{i} J_{i} f \circ \varphi_{i}} \leqslant \frac{\left|\sum_{i} J_{i}^{\prime} f \circ \varphi_{i}\right|}{\sum_{i} J_{i} f \circ \varphi}+\frac{\left|\sum_{i} J_{i}\left(D \varphi_{i}\right)\left(f^{\prime} \circ \varphi_{i}\right)\right|}{\sum_{i} J_{i} f \circ \varphi_{i}} \\
& <\max _{i} \frac{\left|J_{i}^{\prime}\right|}{J_{i}}+\max _{i}\left\|D \varphi_{i}\right\| \frac{\left|f^{\prime} \circ \varphi_{i}\right|}{f \circ \varphi_{i}}
\end{aligned}
$$

where all functions are evaluated at $x \in A$.

From E3 it follows that $\left\|D \varphi_{i}(x)\right\|<1 / \lambda<1$. Also since the partial derivatives of $T$ are uniformly bounded in $B=\left(A \cap T^{-1} A\right)$ we have det $D T(x)$ is uniformly bounded in $B$, so $\operatorname{det} D \varphi_{i}(T(x))=(\operatorname{det} D T(x))^{-1}$ is uniformly 
bounded away from 0 . Therefore there exists $M<\infty$ such that $\sup \left|J_{i}^{\prime}(x)\right| / J_{i}(x) \leqslant M$ where the supremum is taken over all $x$ and all $i$. From inequality (4.3) we obtain:

$$
\operatorname{Reg}(P f) \leqslant M+(1 / \lambda) \operatorname{Reg}(f) .
$$

Iteration of inequality (4.4) yields

$$
\limsup _{n \rightarrow \infty} \operatorname{Reg} P^{n}(f) \leqslant M \lambda /(\lambda-1) \stackrel{\text { def }}{=} \rho
$$

with $\rho$ independent of $f$. The claim is proved.

To obtain a ball which is mapped into itself under $P$, define $H_{\rho}=\{f \in H$ : $\operatorname{Reg} f \leqslant \rho\}$. If $f \in H_{\rho}$ then: $\operatorname{Reg} P f \leqslant M+\rho / \lambda=\rho$, so the set $H_{\rho}$ is invariant under $P$, i.e., $P\left(H_{\rho}\right) \subset H_{\rho}$. We claim that $H_{\rho}$ is a compact convex subset of $C(A)$. First consider compactness. Let $f \in H_{\rho}$. We have assumed that there is some $\beta$ such that any two points $x, y$ in any $A_{i}$ can be joined by a polygonal arc of length at most $\beta$. Applying the inequality $\left|f^{\prime}\right| / f \leqslant \rho$ to each of the straight pieces of the polygonal arc, and then multiplying the results we may obtain

$$
f(y) e^{-\rho \beta}<f(x) \leqslant f(y) e^{\rho \beta} \quad \text { for all } x, y \in A_{i} \text { for all } A_{i} .
$$

In particular $f$ is either identically zero on $A_{i}$ or $\inf _{A_{i}} f>0$. Furthermore

$$
\sup _{A_{i}} f(x) \leqslant e^{\rho \beta} \inf _{A_{i}} f(x)
$$

and $\int_{A} f d m=1$ for all $f \in H_{\rho}$, so $H_{\rho}$ is equibounded. From the inequality $\left|f^{\prime}\right| / f<\rho$ and equiboundedness, it follows that there is a uniform Lipschitz constant for all $f \in H_{\rho} . H_{\rho}$ is closed since the above inequalities must hold for limits of points in $\boldsymbol{H}_{\rho}$.

Clearly $H_{\rho}$ is convex since $\operatorname{Reg} f$ is a convex functional (from Lemma 4.1). From the Schauder Fixed Point Theorem it follows that there exists a function $f^{*} \in H_{\rho}$ such that $P\left(f^{*}\right)=f^{*}$. The measure $\mu$ defined by $d \mu=f^{*} d m$ satisfies conditions (i)-(iii) of Theorem 1.

The fixed point of $P$ does not have to be unique. Also it is possible to have fixed points which are identically zero on some components of $A$. However the theorem below holds. First set

$$
K=\left\{f \in C(A): \inf _{A} f>0 \text { and } \sup _{A} f<\infty \text { and } \int_{A} f=1\right\}
$$

TheOREM 2. Let $T$ satisfy the hypotheses of Theorem 1. Assume in addition, that $T$ is transitive on components. Then there is a unique $f \in K$ such that $P f=f$.

In order to prove this theorem we need the following propositions which may have some independent interest. For these propositions we assume the 
hypotheses of Theorem 1 . We write $\|\cdot\|$ for the supremum norm for $C(A)$.

Proposition 1. Let $f, g \in K$, then the operators $P^{n}, n \geqslant 1$, satisfy

$$
\left\|P^{n}(f)-P^{n}(g)\right\| \leqslant M\|f-g\|
$$

where $M$ depends only on the lower and upper bounds of $f$ and $g$ and not on $n$.

Proof. Let $\beta_{n}(f)=\left\|P_{1}^{n}(f)\right\|_{1}, \beta_{n}(g)=\left\|P_{1}^{n}(g)\right\|_{1}$. Since $f, g \in K$ we have $\inf _{A} f>0$ and $\inf _{A} g>0$ so $\beta_{n}(f)$ and $\beta_{n}(g)$ are positive.

$$
\begin{aligned}
\| P^{n}(f)- & P^{n}(g)\|=\| P_{1}^{n}(f) / \beta_{n}(f)+P_{1}^{n}(g) / \beta_{n}(g) \| \\
< & \left\|\left(P_{1}^{n}(f)-P_{1}^{n}(g)\right) / \beta_{n}(f)\right\| \\
& \quad+\left\|P_{1}^{n}(g)\left(\beta_{n}(f)-\beta_{n}(g)\right) /\left(\beta_{n}(f) \cdot \beta_{n}(g)\right)\right\| \\
< & \|f-g\|\left(\left\|P_{1}^{n}(1) / \beta_{n}(f)\right\|+\left\|P_{1}^{n}(g) \beta_{n}(1) /\left(\beta_{n}(f) \cdot \beta_{n}(g)\right)\right\|\right) \\
& \leqslant\|f-g\|\left(\left\|P^{n}(1) \beta_{n}(1) / \beta_{n}(f)\right\|+\left\|P^{n}(g)\right\| \beta_{n}(1) / \beta_{n}(f)\right) .
\end{aligned}
$$

Since the functions 1 and $g$ are in $H_{\rho}$, from (4.7) it follows that $\sup _{n}\left\|P^{n}(1)\right\|$ $<\infty, \sup _{n}\left\|P^{n}(g)\right\|<\infty$ and

$$
\beta_{n}(1) \inf _{A} f \leqslant \beta_{n}(f) \leqslant \beta_{n}(1) \sup _{A} f \text {. }
$$

Proposition 2. For $f \in K$ with $P f=f$, we have $f \in H_{\rho}$.

Proof. Let $f \in K$ and let $s=\sup _{A} f$ and $\delta=\inf _{A} f$. Let $g$ be Lipschitzean with $\inf _{A} g \geqslant \delta$ and with $\sup _{A} g \leqslant s$. From Proposition 1 it follows that for all $g$ satisfying the above conditions we have

$$
\left\|P^{n}(f)-P^{n}(g)\right\| \leqslant M\|f-g\|
$$

where $M=M(\delta, s)$ depends only on $\delta$ and $s$. Notice that $f$ is a limit of such functions $g$. Let $\varepsilon>0$ be fixed and let $\|f-g\| \leqslant \varepsilon / M$. It follows that $\left\|P^{n} f-P^{n} g\right\|_{\text {def }} M\|f-g\| \leqslant \varepsilon$ for all $n \geqslant 1$. Hence $\left\|f-P^{n} g\right\|<\varepsilon$. The functions $g_{n} \stackrel{\text { def }}{=} P^{n} g$ are Lipschitzean and $\lim \sup _{n \rightarrow \infty} \operatorname{Reg} g_{n}<\rho$. Hence $d\left(g_{n}, H_{\rho}\right) \rightarrow 0$ as $n \rightarrow \infty$ so $d\left(f, H_{\rho}\right) \leqslant \varepsilon$ where $d(x, Y)=\inf \{\|x-y\|:$ $y \in Y\}$. Since this is true for all $\varepsilon>0$ and $H_{\rho}$ is closed, $f$ is in $H_{\rho}$.

We now give a result that concludes the fixed points are not identically zero on any components.

Proposition 3. Assume $T$ is transitive on components. If $f \in H_{\rho}$ and $P f=f$, then $f \in K$.

Proof. Let $f$ be in $H_{\rho}$ with $P f=f$. Suppose that for some $i$, inf $A_{i} f=0$. From inequality (4.7) it follows that $f \equiv 0$ on $A_{i}$. Let $S_{1}$ be the set of components in which $f(x)>0$ and let $S_{2}=A-S_{1}$. Transitivity implies $S_{2} \cap T\left(S_{1}\right)$ is not empty. Let $x_{0} \in S_{2} \cap T\left(S_{1}\right)$. Hence for some $j, \varphi_{j}\left(x_{0}\right) \in$ $S_{1}$ so $f\left(\varphi_{j}\left(x_{0}\right)\right)>0$ and $P f\left(x_{0}\right)>0$. Since $x_{0} \in S_{2}, f\left(x_{0}\right)=0$. This contra- 
dicts the assumption there is an $i$. Regularity and inequality (4.7) now imply $\inf _{A_{i}} f>0$ for all $i$. Since $\left\{A_{i}\right\}$ is finite, $\inf _{A} f>0$.

Proof of THE Theorem 2. Suppose $P$ has two distinct fixed points $f_{1}$ and $f_{2}$ in $K$. From Proposition 2 it follows $f_{1}, f_{2} \in H_{\rho}$. For $j=1,2$ set $B=T^{-1}(A)$ and $\alpha_{j}=\int_{B} f_{j} d m$. Since $\inf _{A} f_{j}(x)>0$, it follows $\alpha_{j}>0$ for $j=1$ and 2 . Consider first the case $\alpha_{1}=\alpha_{2}$. Set

$$
f_{s}(x)=s f_{1}(x)+(1-s) f_{2}(x) \text { for } s \in R \text {. }
$$

We do not restrict $s$ to $[0,1]$. For all $s, \int_{A} f_{s} d m=1$ and if $f_{s}(x)>0$, we have $P f_{s}=f_{s}$. Let $\sigma>1$ be the largest number $s$ for which $\inf _{A} f_{s}>0$. Hence $\inf _{A} f_{\sigma}=0$. Since $f_{\sigma}=\lim _{s \rightarrow \sigma} f_{s}$ we have $f_{\sigma} \in H_{\rho}$ since $H_{\rho}$ is closed. From Proposition $3, f_{\sigma} \in K$ which contradicts $\inf f_{\sigma}=0$. Hence this case $\alpha_{1}=\alpha_{2}$ is vacuous.

Suppose now $\alpha_{1} \neq \alpha_{2}$. Let $\alpha_{1}>\alpha_{2}$. Since $P f=P_{1}(f) / \alpha_{1}$ it follows

$$
P_{1}^{n}\left(f_{1}(x)\right)=\alpha_{1}^{n} f_{1}(x), \quad P_{1}^{n}\left(f_{2}(x)\right)=\alpha_{2}^{n} f_{2}(x) .
$$

Let $\beta$ be such that $\beta f_{2}(x) \geqslant f_{1}(x)$; such a $\beta$ exists since $f_{2}$ is fixed then $\inf _{A} f_{2}>0$. From (4.8) we obtain

$$
\beta \alpha_{2}^{n} f_{2}(x)=\beta P_{1}^{n}\left(f_{2}(x)\right)=P_{1}^{n}\left(\beta \cdot f_{2}(x)\right) \geqslant P_{1}^{n}\left(f_{1}(x)\right)=\alpha_{1}^{n} f_{1}(x)
$$

then $f_{2}(x) \geqslant\left(\alpha_{1} / \alpha_{2}\right)^{n}\left(f_{1}(x) / \beta\right)$ which is absurd since $\left(\alpha_{1} / \alpha_{2}\right)^{n} \rightarrow \infty$.

ExAmple. Let $T:(0,1) \rightarrow R$ be defined by $T(x)=x /(1-x)$. The hypothesis " $\left|T^{\prime}(x)\right| \geqslant \lambda>1$ " is not satisfied since $\inf \left|T^{\prime}(x)\right|=1$. We will show there are no conditionally invariant measures satisfying the conclusions of Theorems 1 and 2 .

Let $\mu$ be an absolutely continuous measure such that $\mu\left(T^{-1}(E)\right)=\alpha \mu(E)$ for all Borel sets $E$, where $\alpha=\mu\left(T^{-1}(0,1)\right)$, Notice that $T^{-1}(1 / n)=1 /(n+$ 1); then $T^{-1}\left(J_{n}\right)=J_{n+1}$ where $J_{n}=\left(n^{-1},(n+1)^{-1}\right)$. We have $J_{n+1}=$ $T^{-n}\left(J_{1}\right)$ hence $\mu\left(J_{n+1}\right)=\mu\left(T^{-n}\left(J_{1}\right)\right)=\alpha^{n} \mu\left(J_{1}\right)$. On the other hand $m\left(J_{n}\right)=$ $n^{-1}-(n+1)^{-1}=n^{-1}(n+1)^{-1}$. Hence $\mu\left(J_{n+1}\right) / m\left(J_{n+1}\right)=\alpha^{n} \mu\left(J_{1}\right)(n+$ $1)(n+2) \rightarrow 0$, so the density is not bounded away from 0 .

5. Convergence. A natural question is whether $P^{n}(f)$ converges to the unique fixed point $f^{*}$ of $P$ for all $f \in K$. We will show by example that this is not the case.

ExAmple. Let $A=(-1,0), A_{2}=(0,1)$ and let $T(x)=-2 x$. Let $0<q<$ 1 and let $f_{q}(x)=q$ for $x \in A_{1}$ and $f_{q}(x)=1-q$ for $x \in A_{2}$. Notice that $f$ is continuous at each point of $A_{1} \cup A_{2}$. We have:

$$
P\left(f_{q}(x)\right)=f_{q}(-x / 2)=f_{q}(-x) \text { for all } x \in A_{1} \cup A_{2} \text {. }
$$

So $P^{2 n}\left(f_{q}(x)\right)=f_{q}(x)$ and $P^{2 n+1}\left(f_{q}(x)\right)=f_{q}(-x)$. Hence $P^{n}\left(f_{q}\right)$ is periodic and does not converge when $q \neq \frac{1}{2}$. This example may be changed slightly. 
Let $T(x)=2 x$ and let $A=(-1,1)$. Define $f_{q}(x)=q$ on $(-1,0)$ and $=1-$ $q$ on $[0,1), q \in(0,1), q \neq \frac{1}{2}$. Each $f_{q}$ is fixed point of $P$. This example shows that the requirement of continuity in Theorem 2 is necessary for uniqueness.

In proving the following propositions we always assume the hypotheses of Theorem 2. We say a function $g$ belongs to the limit set of $f, L(f)$, if there exists a subsequence of $\left\{P^{n}(f)\right\}$ converging to $g$.

Proposition 4. Let $f$ be in $K$ and let $g \in L(f)$. Then $g$ is in $H_{\rho}$.

The proof is omitted since it is similar to that of Proposition 2. We will show now that if $g$ is in the limit of $f, L(f)$, for some $f \in K$, then $g$ is in the limit set of itself.

Proposition 5. Let $f \in K$ and let $g \in L(f)$. Then $g \in L(g)$.

Proof. Let $P^{n_{k}}(f) \rightarrow g$. Then

$$
\left\|P^{n_{k+1}-n_{k}}(g)-g\right\| \leqslant\left\|P^{n_{k+1}-n_{k}}(g)-P^{n_{k+1}-n_{k}}\left(P^{n_{k}}(f)\right)\right\|+\left\|P^{n_{k+1}}(f)-g\right\| \text {. }
$$

From Proposition 2 it follows that:

$$
\left\|P^{n_{k+1}-n_{k}}(g)-P^{n_{k+1}-n_{k}}\left(P^{n_{k}}(f)\right)\right\| \leqslant M\left\|P^{n_{k}}(f)-g\right\|
$$

where $M$ is independent on $n_{k}$. Hence

$$
\left\|P^{n_{k+1}-n_{k}}(g)-g\right\| \leqslant M\left\|P^{n_{k}}(f)-g\right\|+\left\|P^{n_{k+1}}(f)-g\right\| \rightarrow 0 .
$$

It can be proved that if $g \in L(g)$, then $g$ is periodic, but we do not need this fact so we give no details.

Proposition 6. Let $f^{*} \in K$ be the unique fixed point of $P$ and let $g \in K$ be such that $g \in L(g)$, i.e. let $P^{n_{k}}(g) \rightarrow g$. Then $\beta\left(n_{k}\right) / \alpha^{n_{k}} \rightarrow 1$ where $\beta\left(n_{k}\right)=$ $\left\|P_{1}^{n_{k}}(g)\right\|_{1}$ and $\alpha=\left\|P_{1}\left(f^{*}\right)\right\|_{1}$.

Proof. Since $f^{*}$ and $g$ are in $K$, there exist constants $\gamma_{1}, \gamma_{2}$ such that

$$
\gamma_{1} g \leqslant f^{*} \leqslant \gamma_{2} g \text { and } \gamma_{1} g \leqslant 1 \leqslant \gamma_{2} g, \quad 0<\gamma_{1}<\gamma_{2} .
$$

Since the operator $P_{1}$ is linear we have,

$$
\frac{1}{\gamma_{2}} \leqslant \frac{\beta(n)}{\alpha^{n}} \leqslant \frac{1}{\gamma_{1}} \text { for all } n \text {. }
$$

Let $\left\{m_{k}\right\}$ be such that $P^{m_{k}}(g) \rightarrow g$ and $\beta\left(m_{k}\right) / \alpha^{m_{k}}$ is convergent (say to $c$ ). We have:

$$
\begin{aligned}
\frac{\beta\left(2 m_{k}\right)}{\alpha^{2 m_{k}}} & =\frac{\left\|P_{1}^{m_{k}}\left(P_{1}^{m_{k}}(g)\right)\right\|_{1}}{\alpha^{2 m_{k}}}=\frac{\beta\left(m_{k}\right)}{\alpha^{2 m_{k}}}\left\|P_{1}^{m_{k}}\left(P^{m_{k}}(g)\right)\right\|_{1} \\
& =\frac{\beta\left(m_{k}\right)}{\alpha^{m_{k}}}\left\|\frac{P_{1}^{m_{k}}(g)}{\alpha^{m_{k}}}+\frac{P_{1}^{m_{k}}\left(P^{m_{k}}(g)-g\right)}{\alpha^{m_{k}}}\right\|_{1}
\end{aligned}
$$


As $k \rightarrow \infty$ we see that last term above becomes negligible. In fact

$$
\alpha^{-m_{k}}\left\|P_{1}^{m_{k}}\left(P^{m_{k}}(g)-g\right)\right\|_{1} \leqslant\left\|P^{m_{k}}(g)-g\right\| \cdot \alpha^{-m_{k}}\left\|P_{1}^{m_{k}}(1)\right\|_{1}
$$

and the right-hand side tends to 0 since from inequality (5.1) it follows that $\alpha^{-m_{k}}\left\|P_{1}^{m_{k}}(1)\right\|_{1}$ is bounded. Then from the above inequalities we obtain

$$
\lim _{k} \frac{\beta\left(2 m_{k}\right)}{\alpha^{2 m_{k}}}=\lim _{k}\left(\frac{\beta\left(m_{k}\right)}{\alpha^{m_{k}}}\right)^{2} .
$$

With the same procedure we can prove

$$
\lim _{k} \frac{\beta\left(n \cdot m_{k}\right)}{\alpha^{n \cdot m_{k}}}=\lim _{k}\left(\frac{\beta\left(m_{k}\right)}{\alpha^{m_{k}}}\right)^{n} .
$$

Then if $\lim _{k} \beta\left(m_{k}\right) / \alpha^{m_{k}} \neq 1$, this equality contradicts inequality (5.1).

Theorem 3. Let T satisfy the hypotheses of Theorem 2. Assume, in addition, that for all $n, T^{n}$ is transitive on components. Then for all $f \in K, P^{n} f$ converges uniformly to $f^{*}$, the unique fixed point of $P$.

In order to prove this theorem we need the following proposition.

Proposition 7. Let T satisfy the hypotheses of Theorem 3. Then there exists $d>0$ such that, for all $f \in C(A)$,

$$
\liminf _{n \rightarrow \infty}\left(P^{n} f\right)(x) \geqslant d \text { for all } x \in A .
$$

Proof. Since for all $n, T^{n}$ is transitive on components, Condition E2 implies there exists an $N$ such that $F^{N}\left(A_{i}\right) \supset A$ for all $i$. Let $f \in H_{\rho}$ :

$$
P_{T^{N}} f(x)=\sum_{i}\left|\operatorname{det} D \psi_{i}(x)\right| f \circ \psi_{i}(x)
$$

where the $\psi_{i}$ are the inverses of $T^{N}$ at $x$. Since the average value of $f$ is 1 , there is some $A_{j}$ for which $\sup _{A_{j}} f \geqslant 1$. Since $f \in H_{\rho}$, inequality (4.7) implies $\inf _{A_{j}} f \geqslant e^{-\rho \beta}$. For each $x \in A$ there is some $i_{0}$ such that $\psi_{i_{0}}(x) \in A_{j}$. Hence

$$
\begin{aligned}
\left(P_{T^{N}} f\right)(x) & \geqslant\left|\operatorname{det} D \psi_{i_{0}}(x)\right| f\left(\psi_{i_{0}}(x)\right) \geqslant e^{-\rho \beta} \min \left|\operatorname{det} D \psi_{i}(x)\right| \\
& \geqslant e^{-\rho \beta} / \sup _{A}\left|\operatorname{det} D\left(T^{N}\right)\right| \stackrel{\text { def }}{=} d>0 .
\end{aligned}
$$

This number $d$ is independent of $f \in H_{\rho}$.

Proof of Theorem 3. Let $f \in K$ and let $g \in L(f)$; we will show that $g=f^{*}$. From Proposition 4, 5 and 6 it follows that there exists a sequence $m_{k}$ such that as $k \rightarrow \infty$,

$$
\alpha^{-m_{k}} P_{1}^{m_{k}}(g) \rightarrow g
$$

For $s \in R$ define $f_{s}(x)=s f^{*}(x)+(1-s) g(x)$. In particular $\int_{A} f_{s}=1$. Let $J$ be the largest interval containing $[0,1]$ such that if $s \in J$, then $\inf _{A} f_{s}>0$. By 
construction $J$ is open. For $s \in J$,

$$
\begin{aligned}
\alpha^{-m_{k}} P_{1}^{m_{k}}\left(f_{s}\right) & =\alpha^{-m_{k}}\left[s P_{1}^{m_{k}}\left(f^{*}\right)+(1-s) P_{1}^{m_{k}}(g)\right] \\
& \rightarrow s f^{*}+(1-s) g
\end{aligned}
$$

which is $f_{s}$. Since $\left\|f_{s}\right\|_{1}=1$, the norm of the left-hand side converges to 1 . Hence

$$
P_{1}^{m_{k}}\left(f_{s}\right) /\left\|P_{1}^{m_{k}}\left(f_{s}\right)\right\|_{1} \rightarrow f_{s},
$$

so that $f_{s} \in L\left(f_{s}\right)$. From Proposition $4, f_{s}$ is in $H_{\rho}$ for all $s \in J$. If $t$ is a boundary point of $J, f_{t}$ is the limit of points in $H_{\rho}$. Since $H_{\rho}$ is compact, $f_{t} \in H_{\rho}$. Hence $t \in J$. Hence $J$ is also closed, so $J=R$. We now have $f_{s}(x)>0$ for all $s \in R$ so $g=f^{*}$.

6. Applications. (1) The metastable Lorenz map on an interval. This paper was initially motivated by the following: Lorenz [4] studied a system of ordinary differential equations depending on a parameter $r$ (Rayleigh number). In describing the behavior of his system he investigated (for $r=28$ ) a map $T_{r}$ on the unit interval (see [8] for a more detailed discussion.) The graphs of the maps $T_{r}$ for different values of $r$ are shown in Figure 1. The map $T_{r}$ is smooth except for the mid-point and $\inf \left(T_{r}^{\prime}(x)\right)>1$ for each $r$ under investigation. There is a "critical" value $r_{0} \approx 24.06$ such that, for values of $r<r_{0}, T_{r}[0,1] \supset[0,1]$ while, for $r>r_{0}, T_{r}[0,1] \subset[0,1]$. For $r<r_{0}$ our Theorems 1, 2 and 3 can be applied and the behavior of the system can be described in terms of conditionally invariant measures. For $r>r_{0}$ the theorems in [2], [5] apply since $T$ is piecewise expanding. The graph of $T_{r}$ was determined numerically for each parameter $r$ in the following way. Let $(x, y, z)(t)$ be a solution of the Lorenz system. Let $z\left(t_{n}\right)$ be the sequence of successive local maxima of $z(t)$. For large $n$ we have $z\left(t_{n+1}\right)=T_{r}\left(z\left(t_{n}\right)\right)$.

(2) The map $T(x)=a x(1-x)$ for $a=3.83$. Smale and Williams [7] investigated structural stability of this map. For this value of $a$ the map $T$ has a stable periodic orbit of period 3 which is (approximately)

$$
0.1561 \rightarrow 0.5096 \rightarrow 0.9579 \rightarrow 0.1561 \text {. }
$$

$T$ has also an unstable periodic orbit of period 3 which is

$$
0.1635 \rightarrow 0.5290 \rightarrow 0.9552 \rightarrow 0.1635 \text {. }
$$

Let $p=0.1635 \ldots$ be the unstable periodic point of period 3. Let $A_{1}=(p, 1$ - $T(p)), A_{2}=\left(T(p), T^{2}(p)\right)$ and $A=A_{1} \cup A_{2}$. (See Figure 2.) Define $S(x)$ $=T^{6}(x)$. Direct evaluation shows that on $A \cap S^{-1}(A)$, inf $\left|S^{\prime}(x)\right| \approx 1.036$. On the other hand $S\left(A_{1}\right) \subset A, S\left(A_{2}\right) \subset A$ so that $S$ and its powers are transitive on components.

The hypotheses of Theorem 1, 2 and 3 are satisfied by $S$ restricted to $A$. Hence $S$ has a unique conditionally invariant measure with smooth density $f^{*}=P_{S}\left(f^{*}\right)$. From Theorem 3 it follows that $P_{S}^{n}(f) \rightarrow f^{*}$ for all $f \in K$. 


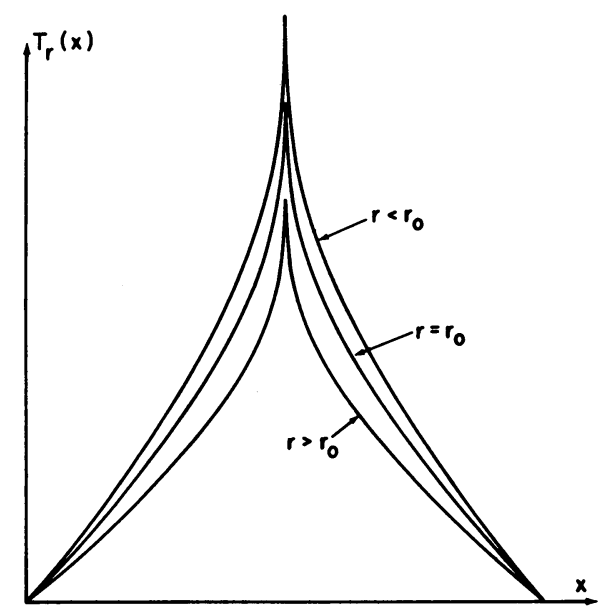

Figure 1

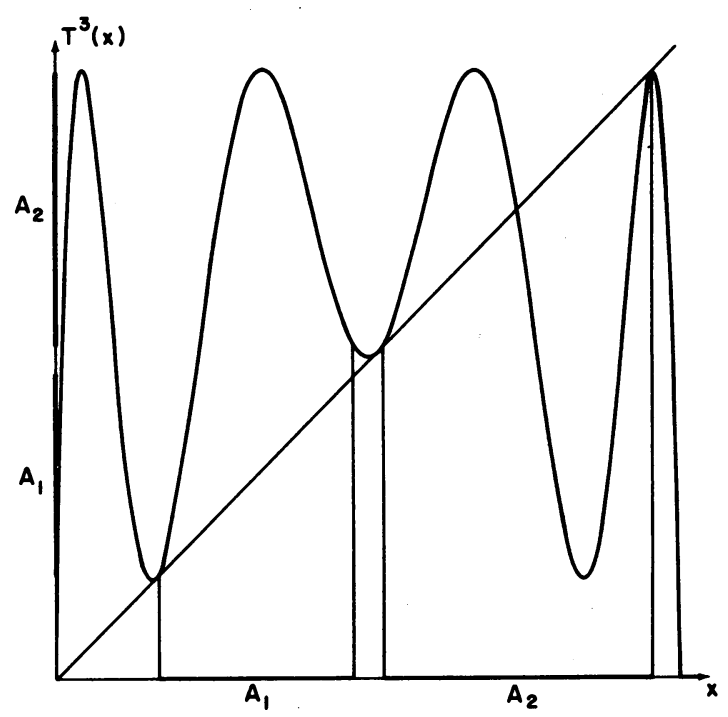

Figure 2

Coming back to $T$ we have $P_{T}^{6}=P_{S}$ then, for all $f \in K$,

$$
P_{T}^{6 n}(f)=P_{S}^{n}(f) \rightarrow f^{*} \text {. }
$$

We claim $f^{*}$ is a fixed point for $P_{T}$ and it is unique and for every $f \in K$, $P_{T}^{n}(f) \rightarrow f^{*}$. For $f \in K$ and $i=0,1, \ldots, 5$ we have $P_{T}^{i}(f) \in K$, so applying (6.1) to $P_{T}^{i}(f)$ instead of $f$ yields $P_{T}^{6 n}\left(P_{T}^{i} f\right)=P_{S}^{n}\left(P_{T}^{i} f\right) \rightarrow f^{*}$. On the other hand $P_{T}^{6 n}\left(P_{T}^{i} f\right)=P_{T}^{i}\left(P_{T}^{6 n} f\right) \rightarrow P_{T}^{i} f^{*}$. Hence $f^{*}=P_{T}^{i}\left(f^{*}\right)$ and $P_{T}^{n}(f) \rightarrow f^{*}$. 
Computer estimates of the $\alpha$ for which $P_{1} f^{*}=\alpha f^{*}$ yield $\alpha \approx .98$. While Smale and Williams investigated the structural stability with emphasis on the attracting period 3 orbit, our point of view is that the decay to a neighborhood of this stable orbit is fairly slow and may be characterized statistically as an exponential decay process.

(3) A continuous version of the von Neumann Middle Square Method. J. von Neumann suggested the following procedure for generating random numbers that appear random. If we want numbers with $2 N$ digits we choose some number with $2 N$ digits and square it (giving in general $4 N$ digits). Our new number is the middle $2 N$ digits. Iterating this process yields a long string of numbers with no apparent regularity unless the generating procedure is known. However, the process can eventually get "caught" in the set of 6 digit numbers beginning with 000 . A continuous analogue discussed by $\mathrm{Li}$ and Yorke $[18]$ is the continuous map on $(0,1)$ defined by

$$
\tau(x)=10^{3} x^{2} \bmod 1 .
$$

Notice 0 is stable with $\tau^{n}(x) \searrow 0$ whenever $x \in\left[0,10^{-3}\right]$. It is easy to verify that with $A=\left[10^{-3}, 1\right], \tau$ satisfies the conditions of our Theorem 3 .

Appendix. In Theorem 1 we stated that if $T$ is in $C^{\infty}(\bar{A})$ then any $\mu$ which satisfies (i)-(iii) has a $C^{\infty}$ density $d \mu / d m$. We now prove this result in two steps, first in the case $A \subset R^{1}$ and then we make comments on how to extend the notation and proof to the general case $A \subset R^{n}$. We have proved in Theorem 1 that

$$
\operatorname{Reg} P f \leqslant M_{10}+\lambda^{-1} \operatorname{Reg} f \text { for all } f \in H
$$

where $M_{10}=M$ depends on $T$ but not on $f$. From the above inequality it follows

$$
\limsup _{j \rightarrow \infty} \operatorname{Reg} P^{j}(f) \leqslant M_{10} \lambda /(\lambda-1) .
$$

Let $T$ be in $C^{\infty}(\bar{A})$ and let $f$ be in $C^{\infty}(A)$. For $n=1,2, \ldots$ define

$$
\operatorname{Reg}^{(n)} f=\sup \left\{\left|f^{(n)}(x)\right| / f(x): x \in A \text { and } f(x)>0\right\} \text {. }
$$

Hence $\operatorname{Reg}^{(1)} f=\operatorname{Reg} f$. We have

$$
\frac{(P f)^{\prime \prime}(x)}{(P f)(x)}=\frac{\Sigma\left|\varphi_{i}^{\prime \prime \prime}\right| f \circ \varphi_{i}+3 \Sigma\left|\varphi_{i}^{\prime}\right|\left|\varphi_{i}^{\prime \prime}\right|^{2} f^{\prime} \circ \varphi_{i}+\Sigma\left|\varphi_{i}^{\prime}\right|^{3} f^{\prime \prime} \circ \varphi_{i}}{\Sigma\left|\varphi_{i}^{\prime}\right| f \circ \varphi_{i}}
$$

where sums are taken over $i$ and where $\varphi_{i}$ and its derivative are evaluated at $x$.

Using Lemma 4.1 we get

$$
\frac{(P f)^{\prime \prime}(x)}{(P f)(x)} \leqslant \max _{i}\left|\frac{\varphi_{i}^{\prime \prime \prime}}{\varphi_{i}}\right|+3\left|\varphi_{i}^{\prime \prime}\right|^{2} \frac{\left|f^{\prime} \circ \varphi_{i}\right|}{f \circ \varphi_{i}}+\left|\varphi_{i}^{\prime}\right|^{2}\left|\frac{f^{\prime \prime} \varphi_{i}}{f \circ \varphi_{i}}\right| \text {. }
$$


Let $M_{20}=\sup _{i, x}\left|\varphi_{i}^{\prime \prime \prime} / \varphi_{i}^{\prime}\right|$ and $M_{21}=3 \sup _{i, x}\left|\varphi_{i}^{\prime}\right|$ and recall $1 / \lambda=\sup _{i, x}\left|\varphi_{i}^{\prime}\right|$. Substituting these constants yields the inequalities

$$
\begin{gathered}
\left|\frac{(P f)^{\prime \prime}(x)}{P f(x)}\right| \leqslant M_{20}+M_{21} \max _{i} \frac{\left|f \circ \varphi_{i}\right|}{f \circ \varphi_{i}}+\lambda^{-2} \max _{i} \frac{\left|f^{\prime \prime} \circ \varphi_{i}\right|}{f \circ \varphi_{i}}, \\
\operatorname{Reg}^{(2)} P f \leqslant M_{20}+M_{21} \operatorname{Reg}^{(1)} f+\lambda^{-2} \operatorname{Reg}^{(2)} f .
\end{gathered}
$$

Notice $M_{20}$ and $M_{21}$ depend on $T$ but not on $f$. Substitute the bound in (A.1) for $\operatorname{Reg}^{(1)} f$ and let

$$
M_{20}^{*}=M_{21}+M_{21} M_{10} \lambda /(\lambda-1)
$$

Then applying (A.2) to $\operatorname{Reg}^{(2)} P^{j}(f)$ we get (just as we obtained inequality (4.5) from inequality (4.4)).

$$
\underset{j \rightarrow \infty}{\lim \sup } \operatorname{Reg}^{(2)} P^{j}(f) \leqslant \rho_{2}
$$

where $\rho_{2}=M_{20}^{*} \lambda^{2} /\left(\lambda^{2}-1\right)$. Analogously we get the inequalities

$$
\operatorname{Reg}^{(n)} P(f) \leqslant M_{n 0}+M_{n 1} \operatorname{Reg}^{(1)} f+M_{n 2} \operatorname{Reg}^{(2)} f+\cdots+\lambda^{-n} \operatorname{Reg}^{(n)} f
$$

and from that follows

$$
\limsup _{j \rightarrow \infty} \operatorname{Reg}^{(n)} P^{j} f(x) \leqslant \rho_{n}
$$

where $\rho_{n}$ is given by some complicated expression. Define $H^{(1)}=H_{\rho}$ and let

$$
H^{(n)}=\left\{f \in C^{\infty}(A): \operatorname{Reg}^{(j)} f \leqslant \rho_{j}, j=1, \ldots, n\right\} .
$$

As was shown for $n=1$ we have: $P\left(H^{(n)}\right) \subset H^{(n)}$ and $\bar{H}^{(n)}$ is compact in $C(A)$ for all $n$. Let $f \in H^{(1)}$ be a fixed point of $P$. Let $\delta_{n}=d\left(f, H^{(n)}\right)$ we want to prove $\delta_{n}=0$. Suppose $\delta_{n}>0$. First assume inf $f>0$. Choose $g \in$ $C^{\infty}$ such that inf $g \geqslant \inf f$ and $|f-g|<\delta_{n} / 2 M$ where $M$ is given by Proposition 1. Then

$$
\begin{aligned}
\delta_{n} & =d\left(f, H^{(n)}\right) \leqslant\left\|f-P^{j} g\right\|+d\left(P^{j} g, H^{(n)}\right) \\
& \leqslant M\|f-g\|+d\left(P^{j} g, H^{(n)}\right) \leqslant \delta_{n} / 2+d\left(P^{j} g, H^{n}\right)
\end{aligned}
$$

which is impossible since $d\left(P^{j}(g), H^{(n)}\right) \rightarrow 0$ as $j \rightarrow \infty$. In other words we can approximate $f$ by a smooth $g$. $P^{j}(g)$ stays near $f$, but also tends asymptotically to $H^{(n)}$. This is only possible if $f \in \bar{H}^{(n)}$. Suppose now $f(x)=0$ for some $x \in A$. Let $A_{+}$be the set of components in which $f(x)>0$. Notice that since $f$ is fixed under $P$, if $f(x)>0$ and $T(x) \in A$ then $f(T(x))>0$ from the definition of $P$. Hence $x \in A_{+}$and $T(x) \in A$ implies $T(x) \in A_{+}$. We may substitute $A_{+}$for $A$ in the statement of Theorem 1; then we can apply our proof to the case " $f>0$ on $A_{+}$". Hence we must only show $f \mid A_{+}$is $C^{\infty}$. Let $g_{k} \in H^{(n)}$ converge to $f$ in $C(A)$. We have $\left|g_{k}^{(n)}(x)\right| / g_{k}(x) \leqslant \rho_{n}$ so $\left|g_{k}^{(n)}(x)\right| \leqslant L \rho_{n}$ where $L$ is a uniform bound for all $g_{k}$. 
Since $g_{k}$ converges to $f, f$ must be at least $C^{n-1}$. Hence $f \in C^{\infty}$ and the proof is complete when $A \subset R^{1}$.

Suppose now $A \subset R^{n}$. For any scalar function $f \in C^{\infty}(A)$ define

$$
f^{(1)}(x)(v)=\left.\frac{d}{d t} f(x+t v)\right|_{t=0}
$$

where $x \in A$ and $v \in R^{n}$ and for $v_{1}, \ldots, v_{s} \in R^{n}$ define

$$
f^{(s)}(x)\left(v_{1}, \ldots, v_{s}\right)=\left.\frac{d}{d t} f^{(s-1)}\left(x+t v_{s}\right)\left(v_{1}, \ldots, v_{s-1}\right)\right|_{t=0} \text {. }
$$

Set

$$
\left\|f^{(s)}(x)\right\|=\left\{\sup f^{(s)}(x)\left(v_{1}, \ldots, v_{s}\right): v_{i} \in R^{n},\left|v_{i}\right|=1, i=1, \ldots, s\right\} .
$$

Let $D \varphi_{i}$ be the Jacobian matrix of $\varphi_{i}$ and let $J_{i}=\left|\operatorname{det} D \varphi_{i}\right|$. Evaluating $P f$ at each $x$ in $A$ we get: $P f=\Sigma_{i} J_{i} \circ \varphi_{i}$. Hence we get

$$
\begin{gathered}
(P f)^{(1)}(v)=\sum_{i} J_{i}^{(1)}(v) f \circ \varphi_{i}+J_{i} f^{(1)} \circ \varphi_{i}\left(D \varphi_{i}, v\right), \\
\operatorname{Reg}^{(1)} P f \leqslant \sup _{i, x} \frac{\left\|J_{i}^{(1)}\right\|}{J_{i}}+\lambda^{-1} \sup _{i, x} \frac{\left\|f^{(1)}\right\|}{f} \leqslant M_{i 0}+\lambda^{-1} \operatorname{Reg}^{(1)} f
\end{gathered}
$$

(using Lemma 4.1). This inequality is analogous to inequality (A.0). In general

$$
(P f)^{(k)}\left(v_{1}, \ldots, v_{k}\right)=\sum_{i}\left(J_{i} \circ \circ \varphi_{i}\right)^{(k)}\left(v_{1}, \ldots, v_{k}\right)
$$

and

$$
\left(J_{i} f \circ \varphi_{i}\right)^{(k)}\left(v_{1}, \ldots, v_{k}\right)=\sum_{j=0}^{k} f^{(j)} \circ \varphi_{i}(\cdot, \ldots, \cdot)
$$

where $f^{(0)}=f$; the unspecified terms in parentheses are expressions containing $D \varphi_{i}$ and its higher derivatives up to order $k_{j}$. For $j=k$ the explicit expression is

$$
f^{(k)} \circ \varphi_{i}\left(\left(D \varphi_{i}\right) v_{1},\left(D \varphi_{i}\right) v_{2}, \ldots,\left(D \varphi_{i}\right) v_{k}\right)
$$

Since by hypotheses $D \varphi_{i}$ and all its derivatives are bounded on $A$, we obtain the following (using the same argument as for computing the $\operatorname{Reg}^{(1)} P f$ inequality):

$$
\operatorname{Reg}^{(k)} P f \leqslant M_{k 0}+M_{k 1} \operatorname{Reg}^{(1)} f+\cdots+M_{k, k-1} \operatorname{Reg}^{(k-1)} f+\lambda^{-k} \operatorname{Reg}^{(k)} f .
$$

From this point the proof is the same as in the one-dimensional case.

ACKNOWLEDGEMENTS. We would like to thank Rufus Bowen for his comments on the exponential decay of the probability of remaining in the set for $n$ iterates. 


\section{REFERENCES}

1. J. Kaplan and J. Yorke, Preturbulence: $A$ regime observed in a fluid flow model of Lorenz (preprint).

2. A. Lasota and J. Yorke, On the existence of invariant measures for piecewise monotonic transformations, Trans. Amer. Math. Soc. 186 (1973), 481-488. MR 49 \$538.

3. T. Y. Li and J. Yorke, Ergodic transformations from an interval into itself, Trans. Amer. Math. Soc. 235 (1978), 183-192.

4. E. Lorenz, Deterministic nonperiodic flow, J. Atmospheric Sci. 20 (1963), 130-141.

5. G. Pianigiani, Existence of invariant measures for piecewise continuous transformations, Ann. Polon. Math. (to appear).

6. K. Robbins, $A$ new approach to subcritical instability and turbulent transitions in a simple dynamo, Math. Proc. Cambridge Philos. Soc. 82 (1977), 309-325.

7. S. Smale and R. W. Williams, The qualitative analysis of a difference equation of population growth, J. Math. Biol. 3 (1976), 1-5.

8. J. Yorke and E. Yorke, Metastable chaos: The transition to sustained chaotic behavior in the Lorenz model (preprint).

9. A. A. Kosjakin and E. A. Sandler, Ergodic properties of a certain class of piecewise smooth transformations of a segment, Izv. Vyš̌. Učebn. Zaved. Matematika 118 (1972), 32-40.

10. A. Avez, Propriétés ergodiques des endomorphismes dilatants des variétés compactes, C. R. Acad. Sci. Paris Sér A-B 266 (1968), A610-A612. MR 37 \#6944.

11. K. Krzyzewski and W. Szlenk, On invariant measures for expanding differentiable mappings, Studia Math. 33 (1969), 83-92. MR 39 \#7067.

12. M. S. Waterman, Some ergodic properties of multidimensional $F$-expansions, $Z$. Wahrscheinlichkeitstheorie und Verw. Gebiete 16 (1970), 77-103. MR 44 \$173.

13. S. Wong, Some metric properties of piecewise monotonic mappings of the unit interval (preprint).

14. R. Bowen and D. Ruelle, The ergodic theory of Axiom A flows, Invent. Math. 29 (1975), 181-202.

15. R. Bowen, Some systems with unique equilibrium states, Math. Systems Theory 8 (1974), 193-202.

16. Equilibrium states and the theory of Anosov diffeomorphisms, Lecture Notes in Math., vol. 470, Springer-Verlag, Berlin and New York, 1975.

17. E. Franco-Sanchez, Flow with unique equilibrium states, Amer. J. Math. 99 (1977), 486-514.

18. T. Y. Li and J. A. Yorke, Ergodic maps on $[0,1]$ and pseudo random number generators, Nonlinear Analysis 2 (1978), 473-481.

Istituto Matematico “U. Dini”, Universita di Firenze, Viale Morgagni 67/A, 50134 Firenze, Italy

Institute for Physical Science and Technology, University of Maryland, College Park, Maryland 20742

Department of Mathematics, University of Maryland, College Park, Maryland 20742 